\title{
Cost Modeling of Wavelength Conversion in Wavelength Routing Optical Networks
}

\author{
Xiaobo $\mathrm{Wu}^{1, \text { a }}$, Zhaohui $\mathrm{Wu}^{1}$, Hong Sun ${ }^{1}$ and Xuna $\mathrm{Miao}^{2, \mathrm{~b}}$ \\ ${ }^{1}$ China Academy of Transportation Sciences, Beijing, 100029, China \\ ${ }^{2}$ Henan University of Economics and Law, Zhengzhou, Henan 450052, China \\ aemail: wuxiaobo1980@163.com, bemail: miaoxuna1227@126.com
}

Keywords: Cost Modeling; Routing and Wavelength Assignment; Optical Networks; Complete Graph

\begin{abstract}
With the increasing demand for network service and rapid development of Wavelength Division Multiplexing (WDM) technologies, Wavelength Routing Optical Networks (WRON) is one of the best ways to realize an all-optical network. Focus on the Routing and Wavelength Assignment (RWA) problem in WRON, a routing cost modeling based on K4 complete graph is proposed for evaluating the cost of loss or delay in wavelength converting process.
\end{abstract}

\section{Introduction}

With the increasing demand for network service and rapid development of Wavelength Division Multiplexing (WDM) technologies, Wavelength Routing Optical Networks (WRON) is one of the best ways to realize an all-optical network because of its inherent characteristics such as high transmission data rate and low interference. The topologies of optical networks are expanding from point-to-point transmission to more complex structure such as rings, star, mesh and so on [1, 2].

WRON which refers to a wavelength for the switching granularity is consisted of the node with wavelength routing function and a series of light paths. Taking into account the wavelength continuity constraint, the key problem of WRON is wavelength assignment and routing cost calculation to each established light path through wavelength conversion. It caught great concern form academic institution and commercial organization in recent years [3-5].

In this paper, Routing and Wavelength Assignment (RWA) problem for a new topology is investigated. Through analyzing wavelength requirements in chain structure and ring structure, the process of wavelength conversion is studied for crossed-rings structure. In order to describe the processes and cost of wavelength conversion, a modeling based on K4 complete graph is presented.

\section{Analysis of wavelength requirements}

The topology of wavelength routing optical networks can be considered as a graph $G=(V, E)$, $V=\left\{v_{i} \mid i=1,2,3, \ldots, n\right\}, E=\left\{e_{i j} \mid v_{i} \in V, v_{j} \in V\right\}$, in which $d\left(v_{i}\right)$ denotes degree of the $v_{i}$ node, $v=|V|=n$ denotes the number of vertices and $\varepsilon=|E|=1 / 2 \sum d\left(v_{i}\right)$ denotes the number of edges. Let $N_{w r}$ is wavelength requirement of edges, let $N_{h}$ is hop count of the shortest path (SP) between any two nodes, and let $N_{m h}$ is the biggest hop count of the SPs between any two nodes. We discuss wavelength requirements in 2 typical optical network topologies respectively in the following, such as chain structure and ring structure, as shown in Fig. 1. 


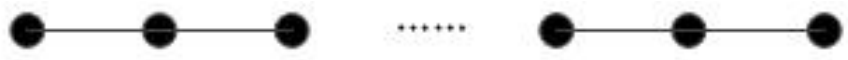

(a) Chain structure

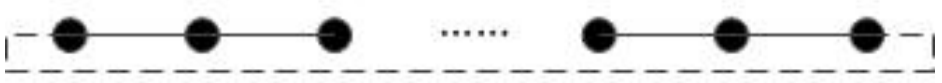

(b) Ring structure

Fig.1. 2 typical optical network topologies

The chain structure is connected graph as shown in Fig. 1. (a), in where the number of vertices $v=|V|=n$, and

$$
\left\{\begin{array}{l}
d\left(v_{i}\right)=1, i=1, n \\
d\left(v_{i}\right)=2, i \neq 1, n
\end{array}\right.
$$

The edges is obtained by calculating the SP between any pair of vertices hop by hop, and the maximum number of SP through these edges is the number of wavelengths to assign [6]. The procedure is as follows:

(1) Find out the SP between any pair of nodes which path is measured by the hop count through the vertex. The connected path between any pair of nodes is SP because there is only one path between two points in chain structure. (2) Calculate the biggest hop count of the SPs between any two nodes $N_{m h}$. $N_{m h}=n-1$, while in chain structure. (3) Wavelength assignment hop by hop.

The situation is called edge overlap that two paths through a common edge. The number of edge overlap in paths with same hop count is $N_{o l}$. There is no edge overlap in any two paths which $N_{h}=1$, and the wavelength requirement is 1 . There is only one edge overlap in any two paths which $N_{h}=2$, and the wavelength requirement is 2 . While $N_{h}=\lfloor n / 2\rfloor$, the wavelength requirement is $\lfloor n / 2\rfloor$. In other words, the wavelength requirement of every path is the hop count of that path in chain structure. The calculation formula of wavelength requirement in chain structure is as follows.

$$
N_{w r}=\left\{\begin{array}{l}
2 \sum_{i=1}^{\frac{n-1}{2}} i=\frac{n^{2}-1}{4}, n \text { is odd. } \\
\frac{n}{2}+2 \sum_{i=1}^{\frac{n}{2}-1} i=\frac{n^{2}}{4}, n \text { is even. }
\end{array}\right.
$$

The ring structure is defined as $C=\left(V_{c}, E_{c}\right)$, in which $V_{c}=\left\{v_{i} \mid i=1,2,3, \ldots, n\right\}$ and $E_{c}=\{(i, i+1)$ $\mid 1 \leqq i \leqq n-1\} \cup\{(n, 1)\}$, as shown in Fig. 1. (b). While the number of vertices $v$ is even, there are 2 SPs in which $N_{m h}=n / 2$. Accordingly, the SPs with the biggest hop count could be assigned only one wavelength to satisfy the wavelength continuity constraint. That is to say, if it existed two SPs with the same biggest hop count, then it could be save wavelength requirement of $n / 2$. The calculation formula of wavelength requirement in ring structure is as follows.

$$
N_{w r}=\left\{\begin{array}{l}
\sum_{i=1}^{\frac{n-1}{2}} i=\frac{n^{2}-1}{8}, n \text { is odd. } \\
\sum_{i=1}^{\frac{n}{2}} i-\frac{n}{4}=\left\lceil\frac{n^{2}}{8}-\frac{1}{2}\right]=\frac{n^{2}}{8}, n \text { is divisible by } 4 . \\
\sum_{i=1}^{\frac{n}{2}} i-\left(\frac{n}{4}-\frac{1}{2}\right)=\frac{n^{2}}{8}+\frac{1}{2}, n \text { is not divisible by } 4 \text { but even. }
\end{array}\right.
$$




\section{Cost modeling of wavelength conversion}

Through the previous analysis of wavelength requirements in chain structure and ring structure, the number of wavelengths less than 16 to satisfy the wavelength continuity constraint while size of ring less than 12. In optical networks topology based on crossed-ring, for example, the network structure is composed of 10 crossed-rings with the size of rings are 8 . In this scene, the wavelength requirements of intra-ring is 8 and the wavelength requirements of inter-ring is 13 . It is obvious that the wavelength routing scheme is feasible whether in intra-ring or in inter-ring. However, the whole network cannot satisfy the wavelength continuity constraint cause of the size of the network is 80 . Therefore, wavelength conversion could be introduced while crossing different rings. As shown in Fig.2. (a), $S_{m, n-1}, S_{m, n}, S_{m, n+1}$ are in an intra-ring, and $S_{m-1, n}, S_{m, n}, S_{m+1, n}$ are in an intra-ring. The resulting 2 all-optical transmissions such as $S_{m, n-1} \rightarrow S_{m, n} \rightarrow S_{m, n+1}$ and $S_{m-1, n} \rightarrow S_{m, n} \rightarrow S_{m+1, n}$ are assigned 2 different wavelengths $\lambda_{i}$ and $\lambda_{j}$. This means that wavelength conversion of $\lambda_{i} \rightarrow \lambda_{j}$ could be implemented based on OE-EO at node $S_{m, n}$ while crossing rings.

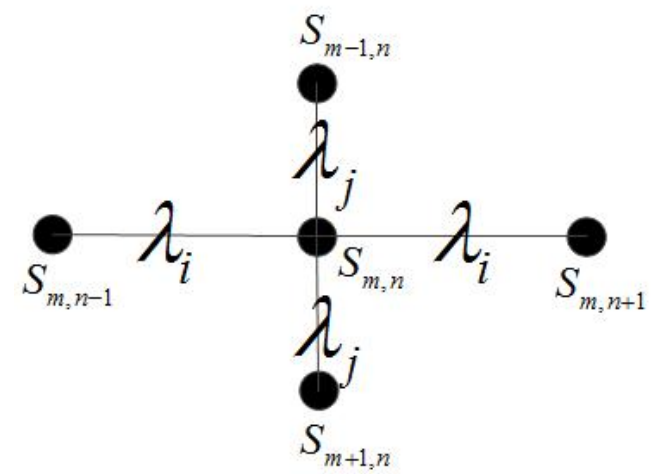

(a) Wavelength conversion in crossed ring network

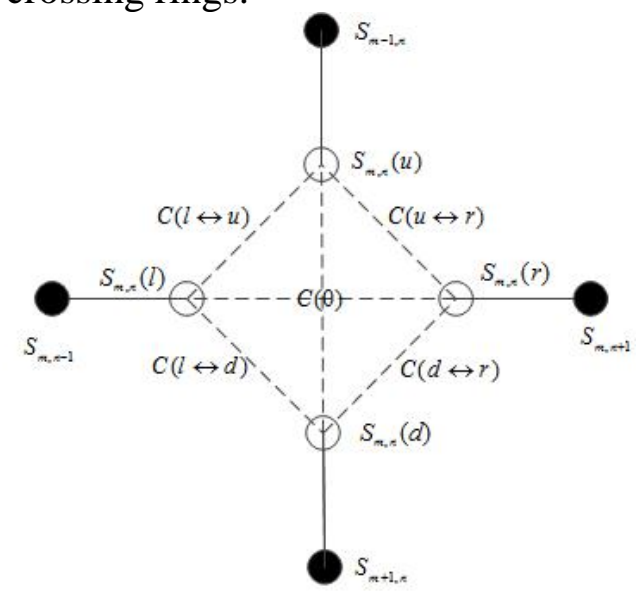

(b) Cost modeling of wavelength Conversion based on $\mathrm{K} 4$ complete graph

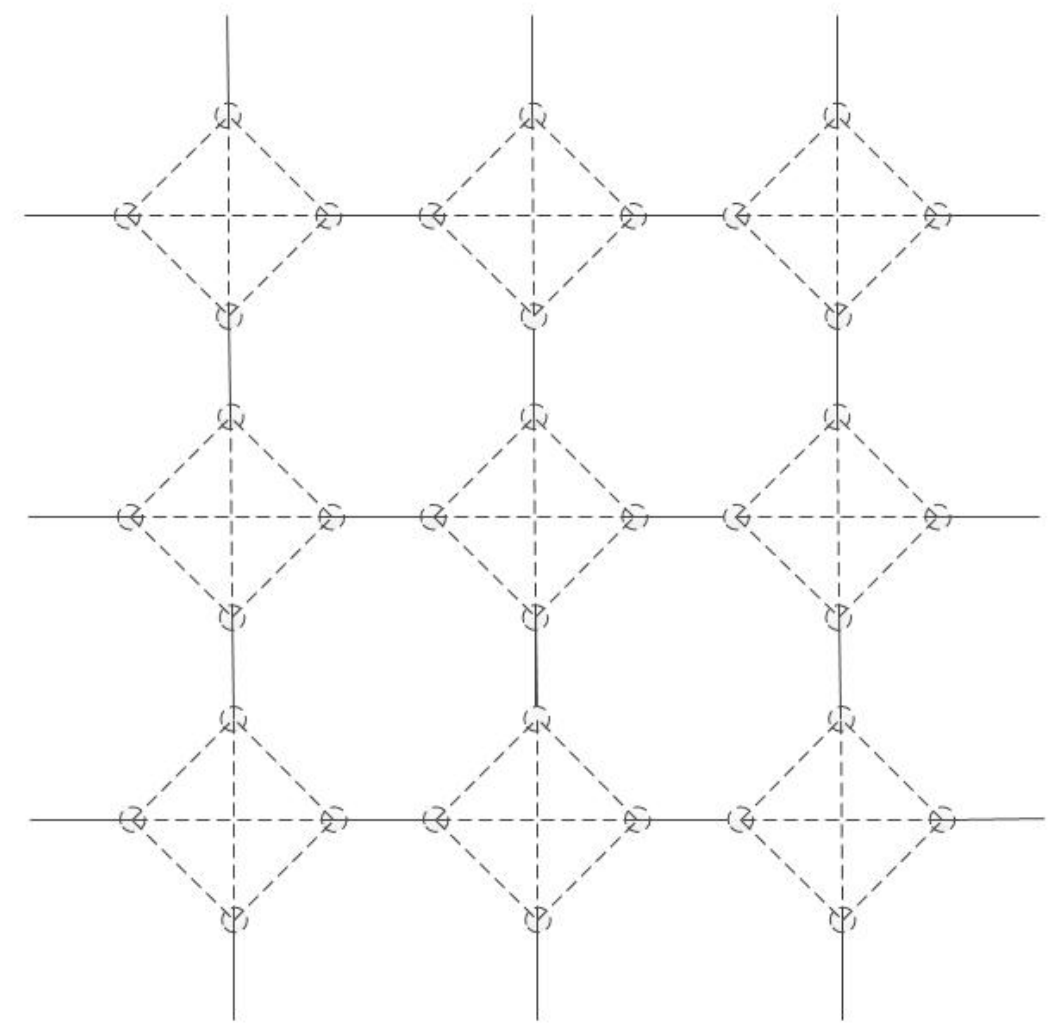

(c) Modeling of Wavelength Routing Optical Networks

Fig.2. Cost modeling of wavelength conversion in crossing ring WRON 
In order to describe the processes and cost of wavelength conversion, a modeling based on K4 complete graph is presented. In this model, the node that is located at the junction of the two rings is abstracted as a K4 complete graph structure which is composed is composed of four virtual nodes. The wavelength conversion function based on OE-EO of the node is to achieve converting on different wavelength assigned on different rings. The cost of wavelength conversion is regarded as the weight of edges to evaluate loss or delay in OE-EO process at the crossing-ring node. As shown in Fig.2. (b), the crossing node $S_{m, n}$ is extended to four virtual nodes as $S_{m, n}(u), S_{m, n}(r), S_{m, n}(d)$ and $S_{m, n}(l)$. K4 complete graph is consisted of these virtual nodes which are connected each other. The edges of each pairs of virtual nodes are assigning the weight of two-way cost functions. Taking into account that OE-EO converting is not occurred in the same ring, the edges, just like $S_{m, n}(u) \leftrightarrow$ $S_{m, n}(d)$, and $S_{m, n}(l) \leftrightarrow S_{m, n}(r)$, are weighting constant $C(0)$. The modeling of whole wavelength routing optical networks topology embedded K4 complete graph is shown in Fig.2. (c).

\section{Conclusion}

Taking into account the wavelength continuity constraint, we mainly discussed routing and wavelength assignment problem in wavelength routing optical networks. Through analyzing wavelength requirements in 2 typical optical network topologies, we analyzed the process of wavelength conversion for crossed-rings structure. In order to describe the cost of wavelength conversion, a modeling based on K4 complete graph is proposed eventually. Some results in this paper provide theoretic reference to research on the routing and transmission $g$ of WRON in the future works.

\section{Acknowledgement}

This work is supported by the National Science Foundation of P. R. China under Grant (No. 61309033, No. 61402147), the Scientific Research Foundation of the Higher Education Institutions of Hebei Province of China (QN20131048) and the Open Fund of the State Key Laboratory of Virtual Reality Technology and Systems (No. BUAA-VR-16KF-02).

\section{References}

[1] Zhang H., JueJ.P. BMukherjee.A Review of Routing and Wavelength Assignment Approaches for Wavelength-Routing Optical WDM Networks [J]. Optical Networks Magazine, 2000 1(1) 47-60.

[2] R. Ramaswami,K. N. Sivarajan. Routing and wavelength assignment in all-optical networks [J]. IEEE/ACM Transaction on Networking, 1995 3(5) 489-500.

[3] Y.Yabin, Z.Xiaoping, Z.Hanyi et al. Choice of the Signal Wavelength in Wavelength Conversion by Cross Gain Modulation in the SOA [J]. CHINAESE JOURNAL OF LASERS, 2002 29(8) 722-728.

[4] Li Wei, He Jun, Li Yuan et al. An Efficient Algorithm for Optimal Allocation of Wavelength Converters in Wavelength Routing Optical Network [J]. Chinese Optics Letters, 2004 2(8) 449-452.

[5] Chu Xiaowen,Li Bo,Zhang Zhensheng.A dynamic RWA algorithm in a wavelength-routed all-optical network with wavelength converters [C]. IEEE. Proceeding of INFOCOM 2003,USA, IEEE, 2003 (3) 1795-1804.

[6] Karafolas N, Baroni S. Optical satellite networks [J]. Journal of Lightwave Technology, 2000 18(12) 1792-1806. 